\title{
Modellierung von Blockiervorgängen in manuellen Kommissioniersystemen mittels Bedientheorie
}

\author{
FURMANS, K.; HUBER, C.; WISSER, J. \\ INSTITUT FÜR FÖRDERTECHNIK UND LOGISTIKSYSTEME, UNIVERSITÄT KARLSRUHE (TH)
}

\begin{abstract}
Aufgrund des hohen Raumnutzungsgrads kommt es in vielen manuellen Kommissioniersystemen, die nach dem Prinzip Mann-zur-Ware organisiert sind, zu Blockiervorgängen und in der Folge zu Produktivitätsverlusten. Zum Einen können sich die Kommissionierer innerhalb der Gänge und Stirngänge nicht überholen, zum Anderen greifen sie zeitgleich auf eine bestimmte Systemressource, z.B. eine Basisstation, zu. In der Literatur wurde diese Problemstellung bisher fast überhaupt nicht berücksichtigt und die wenigen Ansätze, welche diese Problematik erkennen, bieten nur beschränkte Möglichkeiten der Abbildung und Quantifizierung. Aus diesem Grund fördert die Deutsche Forschungsgemeinschaft (DFG) ein Forschungsvorhaben zur Erarbeitung theoretischer Grundlagen, um Blockiervorgänge in manuellen Kommissioniersystemen zu verstehen und deren Auswirkungen in einem analytischen Modell zu beziffern. In diesem Artikel wird die Bedientheorie als eine mögliche Modellierungsmethode identifiziert. Die Übertragung eines manuellen Kommissioniersystems in ein bedientheoretisches Modell wird durchgeführt und auf das Modell anwendbare Lösungsalgorithmen aufgezeigt. Bei der Analyse eines Beispielsystems zeigt sich, dass Blockiervorgänge die Produktivität der einzelnen Kommissionierer senken und die Durchsatzverluste in typischen Betriebsszenarien bis zu $10 \%$ betragen. Für diese Szenarien konnte auch gezeigt werden, dass der relative Fehler zwischen dem bedientheoretischen Modell und einer Simulation unter 4\% liegt. Die Bedientheorie eignet sich folglich als Modellierungsmethode, da sie die Ergebnisse im Vergleich zur Simulation einfach und mit geringem Zeitaufwand berechnet.
\end{abstract}

\section{Einführung}

Kommissioniersysteme sind ein zentraler Bestandteil intralogistischer Systeme. Betrachtet man die Vielzahl möglicher Organisationsformen und die darauf aufbauenden Kommissionierkonzepte ist festzustellen, dass manuelle Kommissionierung häufig nach dem Prinzip „Mann zur Ware“ erfolgt. Reale Datenerhebungen im Rahmen der Warehouse Excellence Studie ergaben, dass 80 \% der Kosten in Systemen anfallen, die nach diesem Prinzip organisiert sind (DCRM 2008).

In manuellen Kommissioniersystemen mit statischer Artikelbereitstellung sind außer der produktiven Arbeitszeit der Kommissionierer auch Wartephasen zu beobachten. Die ungleichmäßige Auslastung verschiedener Bereiche ist hierfür eine mögliche Ursache. Ein weiterer Grund, der in Praxis und Wissenschaft bislang wenig Beachtung findet, sind Blockiervorgänge. Sie entstehen, wenn ein Kommissionierer während der Auftragsbearbeitung durch einen anderen Kommissionierer unterbrochen bzw. behindert wird. Blockiervorgänge können an der Basisstation oder in Gängen auftreten. In Gängen und Stirngängen ist Überholen oder Passieren anderer Kommissionierer aufgrund eines geringen Platzangebots häufig erschwert oder gar unmöglich. Blockiervorgänge resultieren in erhöhten Durchlaufzeiten und mindern die Produktivität bzw. die individuelle Kommissionierleistung und damit letztlich den möglichen Systemdurchsatz. Ein geringes Platzangebot als Auslöser von Blockiervorgängen ist dabei direkte Folge eines durchaus erwünschten, hohen Raumnutzungsgrades. Dieser wird aufgrund zunehmender Variantenvielfalt und des daraus resultierenden, wachsenden Bedarfs an Lagerplatz sowie steigender Energiekosten für Unternehmen auch in Zukunft von Bedeutung sein.

Die einschlägige Fachliteratur setzt sich häufig mit der statischen Leistungsanalyse manueller Kommissioniersysteme auseinander. So berechneten Kunder und Gudehus (Kun-75) in der grundlegenden Arbeit auf diesem Gebiet mittels statischer Betrachtung die mittleren Wegzeiten eines Kommissionierers. Darauf aufbauend entwickelte Hall (Hall-93) Approximationen für die Weglänge. Ratliff und Rosenthal (Rat-83) transformierten zur Optimierung der Kommissionierbewegung die Layouts verschiedener Kommissioniersysteme in Graphen, um ein modifiziertes Travelling-Salesman-Problem (TSP) darauf anwenden zu können. Roodbergen und de Koster (Rood-01) kombinierten den TSP-Ansatz mit einer Sensitivitätsanalyse, um Abkürzungen der Kommissionierer zu integrieren. 
Bei der Planung und Organisation von Kommissioniersystemen wird vorwiegend auf Berechnungsmethoden zurückgegriffen, die auf statischer Berechnung und Leistungsquantifizierung beruhen. Die Ergebnisse dieser Modelle lassen sich in standardisierte Leistungskenngrößen, wie zum Beispiel Anzahl kommissionierter Aufträge pro Stunde oder Anzahl kommissionierter Auftragspositionen pro Stunde umrechnen. Ausgehend von der Leistungskennzahl eines Kommissionierers wird linear auf ein System mit mehreren Kommissionierern geschlossen.

Die Problematik dieser Vorgehensweise verdeutlicht die Diskrepanz des Verlaufes der Durchsatzkurve bei statischer Betrachtung im Vergleich zum angenommenen tatsächlichen Verlauf, der dynamische Effekte berücksichtigt:

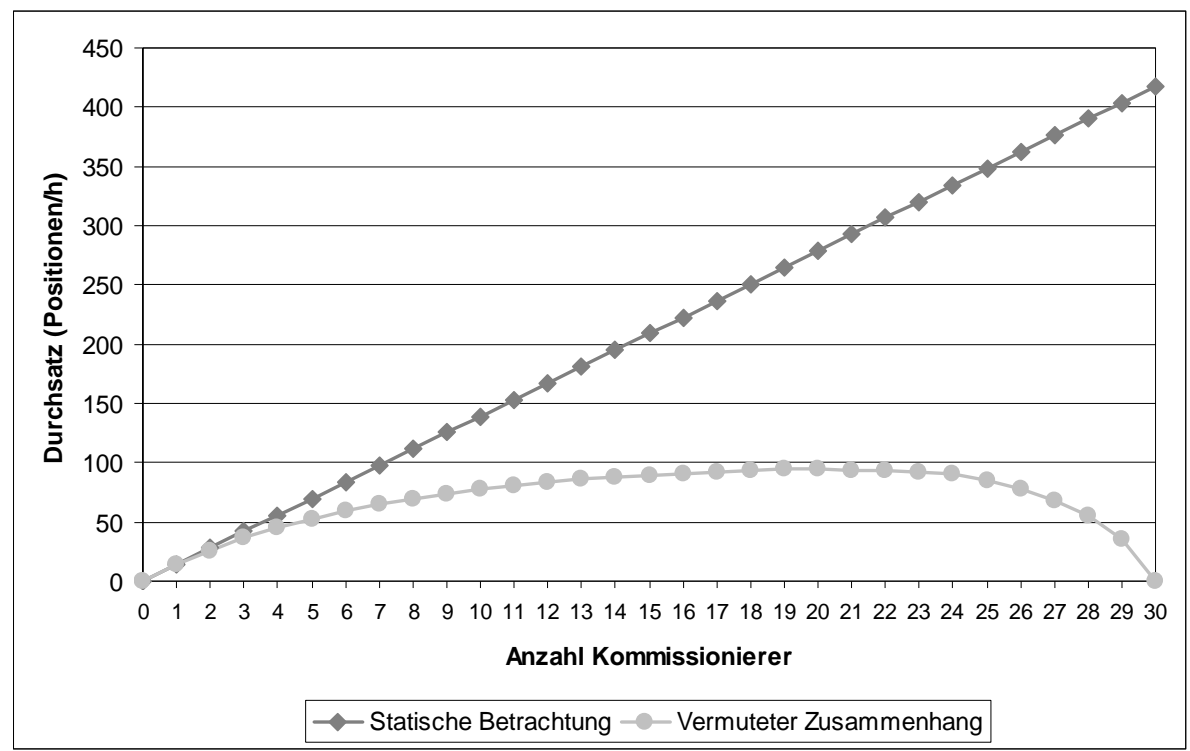

Abbildung 1: Zusammenhang Anzahl der Kommissionierer - Durchsatz

Einen ersten Hinweis auf mögliche Leistungsminderungen geben Ruben und Jacobs (Rub-99), Faißt und Lippolt (Fai-02) sowie Arnold (Arn-03). Sie zweifeln den linearen Verlauf der Durchsatzkurve an und stellen fest, dass eine gegenseitige Behinderung der Kommissionierer stattfindet. Durchsatzverluste in manuellen Kommissioniersystemen durch die steigende Anzahl der Mitarbeiter zu erklären, machte sich Lüning (Lün-05) in seiner Dissertation zur Aufgabe. Er unterscheidet Blockierungen durch zeitgleichen Zugriff auf eine Regalspalte und Behinderungen bei der Fortbewegung innerhalb eines Regalganges. Durch Anwendung der Wahrscheinlichkeitsrechnung und der Kombinatorik ermittelte er einen prozentualen Verlustanteil, der vom statischen Referenzwert abzuziehen ist. Dabei wird jedoch kein vollständiges Kommissioniersystem, sondern lediglich eine isolierte Regalspalte untersucht. Folglich werden keine Bewegungsstrategien abgebildet und es kommt auch nicht zur Bildung von Warteschlangen innerhalb eines Ganges. Eine weitere Arbeit zur Bestimmung der Blockieranteile in Kommissioniersystemen lieferten Gue, Meller und Skufca (Gue-06) mit dem Congestion Model. Sie transformieren die Laufwege der Kommissionierer in geschlossene Kreisbahnen ohne Überholmöglichkeit. Auf Basis zweier fester Verhältnisse zwischen der tatsächlichen Entnahmezeit und der Wegzeit sowie der Wahrscheinlichkeit einer Entnahme leiten die Autoren einen Blockierzeitanteil her. Die getroffenen Modellannahmen ermöglichen jedoch lediglich die Untersuchung sehr weniger Organisationsformen hinsichtlich ihrer Blockierverluste. Darüber hinaus sind die gewählten Verhältnisse nicht realitätsnah.

Aufgrund der mangelnden Berücksichtigung von Blockiervorgängen in Theorie und Praxis wird das Forschungsvorhaben „Blockiervorgänge in manuellen Kommissioniersystemen mit statischer Artikelbereitstellung“ von der Deutschen Forschungsgemeinschaft (DFG) gefördert. Ziel ist die Entwicklung theoretischer Grundlagen zur Beschreibung und Quantifizierung von Blockiervorgängen, um ein besseres Verständnis für die daraus resultierenden Produktivitätsverluste zu gewinnen. Weiterhin werden Strategien zur leistungsfähigen Kommissionierung unter Berücksichtigung von Blockiervorgängen erarbeitet.

Der vorliegende Artikel charakterisiert verschiedene Arten von Blockiervorgängen zunächst grundlegend. Zur Übertragung in ein bedientheoretisches Modell muss aufgezeigt werden, wie die Parameter eines Kommissioniersystems mit Hilfe der Bedientheorie modellierbar sind. Anschließend werden bedientheoretische Lösungsalgorithmen und deren Einsetzbarkeit aufgezeigt. Der bedientheoretischen Berechnung wird abschließend ein Simulationsmodell gegenübergestellt, um die Anwendung der Bedientheorie zu legitimieren. 


\section{Charakteristika von Blockiervorgängen}

Die Quantifizierung von Leistungsminderungen durch Blockierungen in manuellen Kommissioniersystemen erfordert zunächst eine Bestimmung der Zustände, die als Blockierung zu sehen sind. Blockiervorgänge resultieren grundsätzlich in Wartezeiten. Wartezeiten können jedoch auch ohne Blockierungen auftreten. So muss ein Kommissionierer beispielsweise nach Abgabe seines Auftrages an der Basisstation auf den nächsten Auftrag warten. Blockiervorgänge lassen sich von derartigen Wartesituationen wie folgt abgrenzen:

\section{Definition: Ein Zustand heißt Blockiervorgang, wenn die Tätigkeiten eines Kommissionierers durch die Tätigkeiten eines anderen Kommissionierers unterbrochen werden.}

In manuellen Kommissioniersystemen treten Blockiervorgänge immer dann auf, wenn zwei oder mehr Kommissionierer gleichzeitig eine der folgenden Ressourcen nutzen wollen:

- $\quad$ eine Regalspalte im Gang und der zugehörige Platz vor der Regalspalte

- $\quad$ einen Platz des Stirngangs

- $\quad$ eine Basisstation und der zugehörige Platz vor der Basisstation

\subsection{Blockiervorgänge in Gängen und Stirngängen}

Die Gänge und Stirngänge eines manuellen Kommissioniersystems setzen sich aus einer bestimmten Anzahl Einzelplätze zusammen. Ein Einzelplatz ist ein Abschnitt eines Gangs oder Stirngangs, beispielsweise der Platz vor zwei gegenüberliegenden Regalspalten. Er ist $\mathrm{zu}$ einem beliebigen Zeitpunkt nur von einem Kommissionierer nutzbar, wenn die Gangbreite ein erforderliches Mindestmaß unterschreitet. Dann kann es zu Blockiervorgängen kommen, wenn der Einzelplatz durch einen Kommissionierer belegt ist und ein anderer Kommissionierer diesen zeitgleich für einen Greifvorgang benötigt oder passieren muss. In Abhängigkeit der Bewegungsrichtung der Kommissionierer kann es zu einfachen bis komplexen Blockiersituationen kommen (siehe Abbildung 2).
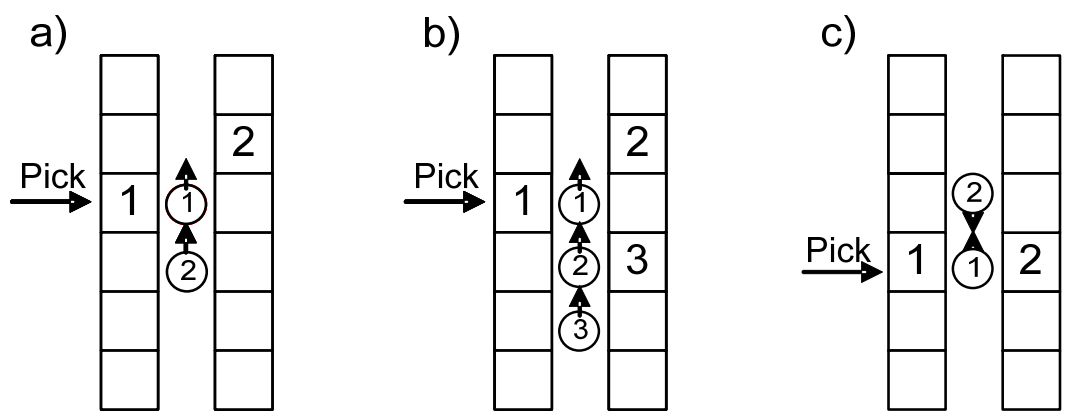

Abbildung 2: Beispielhafte Blockiersituationen

Es sei angenommen, dass die Bewegungsrichtung der Kommissionierer in den Fällen a) und b) jeweils fest und in Beispiel c) beliebig ist.

Wenn Kommissionierer 1 im Fall a) die aktuelle Position seines Auftrags bearbeitet und dabei den gesamten Einzelplatz vor Regalspalte 1 in Anspruch nimmt, kann es zu einem einfachen Blockiervorgang kommen. Kommissionierer 2 wird beim Durchlaufen des Ganges in Richtung des Bereitstellplatzes seiner nächsten Auftragsposition (Regalspalte 2) blockiert. Kommissionierer 2 muss warten, bis Kommissionierer 1 seine Auftragsposition vollständig bearbeitet hat und an Regalspalte 2 vorbei zu seiner nächsten Entnahmeposition läuft.

Im Fall b) tritt zunächst die gleiche Situation ein wie in Fall a). Während Kommissionierer 2 durch Kommissionierer 1 blockiert wird, nimmt er den Einzelplatz vor Regalspalte 3 in Anspruch. Folglich wird Kommissionierer 3 durch Kommissionierer 2 blockiert. In diesem Fall behindert also ein bereits blockierter Kommissionierer einen weiteren Kommissionierer und es kommt $\mathrm{zu}$ einem sogenannten zweifachen Blockiervorgang. Entspricht k der Anzahl der Kommissionierer im System, kann grundsätzlich ein (k-1)-facher Blockiervorgang mit einer Blockierschlange der Länge (k-1) entstehen. Die Auflösung des Blockiervorgangs verläuft bei gleicher Bewegungsrichtung stets nach dem First-Come-First-Serve Prinzip. Vergleichbar mit einer einfachen Warteschlange muss der letzte ankommende Kommissionierer warten, bis alle vor ihm stehenden Kommissionierer abgefertigt wurden. 
Im Fall c) können Blockiervorgänge aus fehlenden Einbahnstraßenregelungen resultieren. Im Beispiel pickt Kommissionierer 1 aus Regalspalte 1, belegt den zugehörigen Einzelplatz und muss nach Abschluss der Entnahme lt. Pfeilrichtung nach oben weitergehen. Kommissionierer 2 muss auf die gegenüberliegende Regalspalte zugreifen und wird durch Kommissionierer 1 blockiert. Es liegt zunächst ein einfacher Blockiervorgang vor (1 blockt 2). Hat Kommissionierer 1 seinen Greifvorgang abgeschlossen, wird er aufgrund der entgegengesetzten Bewegungsrichtung zu Kommissionierer 2 von diesem am weitergehen gehindert (2 blockt 1). Die Auflösung eines solchen zweifach entgegengesetzten Blockiervorgangs ist komplizierter als in den Fällen a) und b). Sie erfordert feste Prioritätsregeln, denn mindestens einer der beiden Kommissionierer muss sich gegen seine ursprüngliche Laufrichtung bewegen, um einen Deadlock zu vermeiden.

\subsection{Blockiervorgänge an der Basisstation}

An der Basisstation eines manuellen Kommissioniersystems werden Auftragslisten und Kommissionierleergut bereitgestellt. Nach der vollständigen Auftragsbearbeitung werden die Kommissionierbehälter an der Basisstation abgegeben. Wie in den Gängen und Stirngängen nimmt ein Kommissionierer die Ressource Basisstation und den zugehörigen Einzelplatz für einen bestimmten Zeitraum in Anspruch. Benötigt ein zweiter oder k-ter Kommissionierer die Ressource zeitgleich, muss er bzw. müssen alle (k-1) Kommissionierer warten. Die Komplexität derartiger Blockiersituationen ist begrenzt und die Auflösung durch eine einfache FIFOAbarbeitung möglich.

\section{Parameter eines manuellen Kommissioniersystems}

Der Aufbau eines manuellen Kommissioniersystems wird durch die Anordnung der Ressourcen bestimmt. Mehrere Gänge, die durch eine feste Anzahl gegenüberliegender Regalspalten charakterisiert sind, werden durch Stirngänge miteinander verbunden. In den Gängen erfolgt die Entnahme der entsprechenden Artikel aus den Regalspalten. Das typische Blocklayout wird um eine Basisstation ergänzt. Das manuelle Kommissioniersystem stellt hier eine abgeschlossene Zone dar (siehe Abbildung 3). Die Anzahl der Gänge und der Regalspalten, sowie Anzahl und Lage der Basisstationen stellen die strukturbestimmenden Parameter dar.

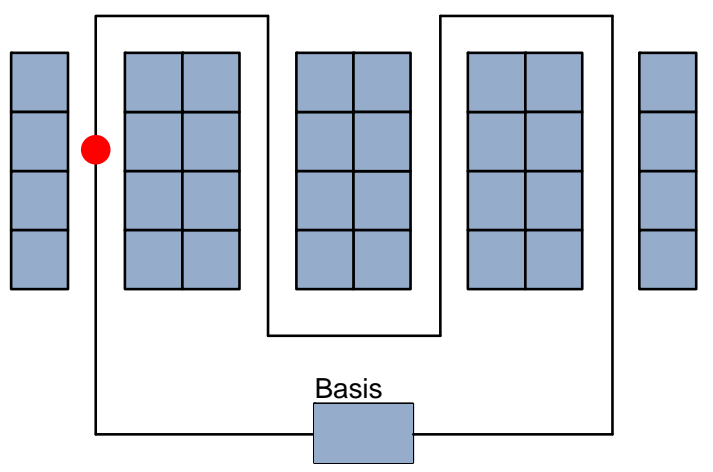

Abbildung 3: Beispielhaftes Kommissioniersystem nach dem Prinzip Mann-zur-Ware

Neben dem physischen Aufbau wird das Kommissioniersystem durch seine Abläufe charakterisiert. Die Anzahl der Kommissionierer ist ein leistungsbestimmender Parameter und wesentlicher Treiber zur Erhöhung des Auftragsdurchsatzes. Daneben hängt die Leistung eines Kommissioniersystems direkt von den Zeiten ab, die an den jeweiligen Ressourcen benötigt werden. Zu berücksichtigen sind die an der Basisstation benötigten Zeiten, die Tot- und Greifzeiten an den jeweiligen Regalspalten sowie die Wegzeiten zum Passieren der Regalspalten. Basiszeiten werden durch die Gestaltung und Organisation der Basisstation bestimmt. Totzeiten lassen sich durch Hilfsmittel zur Unterstützung des Kommissionierers beim Suchen oder Quittieren einer Auftragsposition reduzieren. Die Greifzeiten hängen zum Einen von auftragsspezifischen Entnahmemengen, zum Anderen von Gewicht und Volumen der Artikel ab. Die Wegzeiten ergeben sich aus der Geschwindigkeit der Kommissionierer sowie aus deren Laufwegen. Diese werden durch das Routing der Kommissionierer festgelegt. Die Bewegungsstrategien steuern die möglichen Laufwege und bestimmen die Arten der Blockierungen, die dabei auftreten können (vgl. Abbildung 2). Auftragsspezifische Daten wie die Anzahl der Positionen oder artikelspezifische Daten, wie die Verteilung der Artikel auf die Gänge, haben ebenfalls Einfluss auf das Routing der Kommissionierer. 


\section{Bedientheoretische Modellierung eines manuellen Kommissioniersystems}

Kommissioniersysteme beinhalten zahlreiche stochastische Elemente. Kundenaufträge werden über den Tag typischerweise nicht gleichverteilt an das System übermittelt, sondern sind durch Eingangsspitzen und -tiefen charakterisiert. Somit muss nicht nur der Mittelwert, sondern auch die Standardabweichung betrachtet werden. Gleiches gilt für Bearbeitungszeiten, Auftragsgrößen etc. Auf Basis verschiedener Eingangsparameter berechnet die Bedientheorie Kennwerte eines Systems, beispielsweise die mittlere Durchlaufzeit oder die mittlere Länge einer Warteschlange. Sie findet auf zahlreichen Gebieten Anwendung und hat gegenüber statischen Analysemethoden den Vorteil, dass die Eingangsparameter statistische Größen sind. Sie trägt insbesondere stochastischen Einflüssen eines Systems Rechnung. Eine ausführliche Auseinandersetzung mit den Grundlagen der Bedientheorie findet sich beispielsweise bei Bolch (Bol-98).

Bei Übertragung eines manuellen Kommissioniersystems in ein bedientheoretisches Modell müssen die einzelnen Parameter des Kommissioniersystems (Anzahl der Kommissionierer, Ressourcen, Zeiten und Routings) in die Eingangsparameter des bedientheoretischen Modells (Anzahl der Kunden k, Anzahl der Bediensysteme $\mathrm{n}$, Kapazitäten der Bediensysteme $\mathrm{m}_{\mathrm{i}}$, Bedienzeiten $\mu_{\mathrm{i}}$, Variabilitäten $\mathrm{c}^{2}{ }_{\mathrm{i}}$ und Übergangswahrscheinlichkeiten $\mathrm{q}_{\mathrm{ij}}$ ) übertragen werden.

\subsection{Abbildung eines manuellen Kommissioniersystems auf ein Bediensystemnetzwerk}

\subsubsection{Modellierung der Anzahl Kommissionierer und Wahl des Netzwerktyps}

Die Anzahl der Mitarbeiter wird im bedientheoretischen Modell durch die Anzahl der Kunden im System k dargestellt. In der Praxis ist die Mitarbeiterzahl in Kommissioniersystemen innerhalb eines bestimmten Zeitintervalls in der Regel fix. Sie erhöht sich in Stosszeiten, bleibt zur Bewältigung der Auftragslast in der Folge jedoch auf einem höheren Niveau bestehen.

Aufgrund der Annahme einer fixen Anzahl Kunden entspricht das geschlossene Bediensystemnetzwerk dem realen System am Ehesten. Die resultierenden Durchsätze $\lambda$ (= bearbeitete Aufträge) sind variabel und zustandsabhängig. Wenn sich beispielsweise alle k Kommissionierer in einem einzigen Bediensystem i befinden, so ist die zustandsabhängige Ankunftsrate dieses Systems $\lambda_{\mathrm{i}}(\mathrm{k})=0$. Der Modellierung eines Kommissioniersystems als geschlossenes Bediensystemnetzwerk liegt die Annahme zugrunde, dass an der Basisstation immer ausreichend Aufträge vorhanden sind.

Offene Bediensystemnetzwerke werden an dieser Stelle als weniger geeignet erachtet, da die Durchsatzgröße $\lambda$ in diesen Netzwerken den bearbeiteten Aufträgen entspricht und konstant ist. Die Anzahl der Kommissionierer (= Kunden im System) hingegen ist variabel. Dies deckt sich nicht mit der Annahme fixer Mitarbeiterzahlen.

\subsubsection{Modellierung der Ressourcen}

Der Abbildung der Ressourcen erfolgt anhand der Einzelplatz-Logik. Jeder potentielle Aufenthaltsort eines Kommissionierers im System (Platz vor einer Regalspalte, Platz eines Stirngangs, Platz vor der Basisstation) wird als Einzelplatz definiert und kann stets von nur einem Kommissionierer besetzt sein. Jeder Einzelplatz kann als elementares Bediensystem modelliert werden. Ein Kommissionierer (=Kunde) betritt den Einzelplatz (=Bediensystem), muss diesen entweder Passieren oder je nach Art der Ressource eine Entnahme tätigen oder den Auftrag abgeben (=Bedienzeit) und betritt den nachfolgenden Einzelplatz. Die Summe aller Einzelplätze entspricht der Anzahl Bediensysteme n. Durch die Vernetzung der Bediensysteme lässt sich das Kommissioniersystem dann als geschlossenes Bediensystemnetzwerk modellieren.

\section{Modellierung der Regalspalten}

Innerhalb eines Ganges entspricht ein Einzelplatz dem Platz vor zwei gegenüberliegenden Regalspalten. Jeder Einzelplatz ist ein Bediensystem mit einer Bedienstation und einem Warteraum der Größe 0. Ein Gang wird als Aneinanderreihung mehrerer Bediensysteme modelliert. Die Annahme des nicht vorhandenen Warteraums verhindert, dass sich der Warteraum mit der Bedienstation einer vorgelagerten Regalspalte überschneidet. Bildet man die Regalspalte $n$ mit einer Bedienstation $a b$, ist $n$ wiederum der Warteraum (mit der Kapazität 1) der Nachfolgerbedienstation $(n+1)$. Entsprechend ist die Vorgängerbedienstation ( $n-1)$ der Warteraum (mit der Kapazität 1) der Regalspalte n. 


\section{Modellierung der Stirngänge}

In Abhängigkeit der Länge kann ein Stirngang entweder einen oder mehrere Kommissionierer aufnehmen. Der Stirngang lässt sich dann mit Hilfe eines Einzelplatzes oder als Anreihung mehrerer Einzelplätze modellieren. Die Einzelplätze werden jeweils als elementare Bediensysteme $($ Warteraum $=0)$ abgebildet.

\section{Modellierung der Basisstation}

Die Basisstation sowie die Wege „von und zur“ Basis sind ebenfalls Einzelplätze und werden jeweils als elementares Bediensystem modelliert. Jeder Einzelplatz stellt wiederum den Warteraum des nachgelagerten Einzelplatzes dar. Alle Bediensysteme werden jeweils als Bediensystem mit einer Station ohne Warteraum abgebildet.

Das in Abbildung 3 gezeigte exemplarische Kommissioniersystem lässt sich mit Hilfe von einzelnen Bediensystemen (Kapazität $\mathrm{m}_{\mathrm{i}}=1$ ) als geschlossenes Bediensystemnetzwerk wie folgt abbilden:

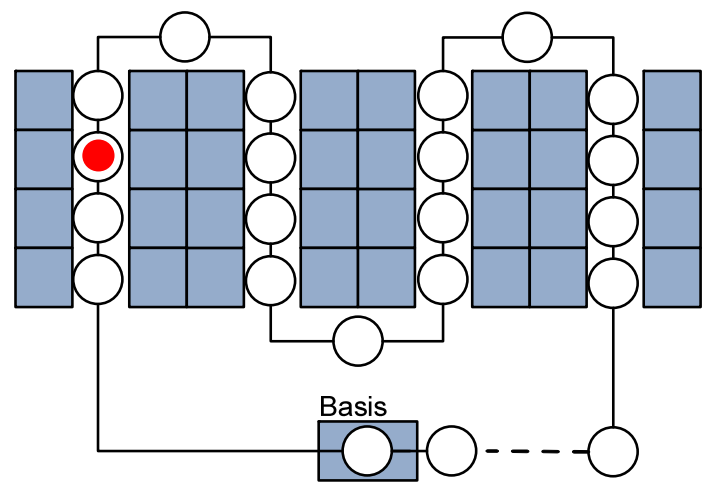

Abbildung 4: Kommissioniersystem als geschlossenes Bediensystemnetzwerk

\subsubsection{Modellierung der Zeiten}

Je nach Art der Ressource müssen die Kommissionierer an den jeweiligen Einzelplätzen unterschiedliche Tätigkeiten durchführen. Die Zeiten, die dafür benötigt werden, müssen in den Bedienzeiten der zugehörigen Bediensysteme berücksichtigt werden. Die Wegzeit muss immer in der Bedienzeit enthalten sein, da jedes Bediensystem einen Einzelplatz und damit eine zu überbrückende Wegstrecke darstellt. Geht man von einer konstanten Geschwindigkeit der Kommissionierer aus, kann die Wegzeit als deterministische Variable angenommen werden. In den Stirngängen kommen keine weiteren Zeiten hinzu und die Bedienzeit entspricht der Wegzeit. Die zur Abgabe eines alten und Aufnahme eines neuen Auftrags benötigte Basiszeit fließt zusätzlich zur Wegzeit direkt in die Bedienzeit der Basisstation ein. Die Anzahl der Auftragspositionen und die Lagerorte der jeweiligen Artikel legen fest, mit welcher Wahrscheinlichkeit ein Kommissionierer an einer Regalspalte eine Entnahme tätigen muss. So kann es Regalspalten geben, die aufgrund der erhöhten Umschlagshäufigkeit der gelagerten Artikel (z.B. Schnelldreher) eine erhöhte Zugriffswahrscheinlichkeit besitzen. Die Bedienzeit der Bedienstation einer Regalspalte muss also mit einer bestimmten Wahrscheinlichkeit zusätzlich die Tot- und Greifzeit beinhalten. Die Greifzeit steigt mit den artikelspezifischen Eigenschaften (Gewicht, Volumen) und der Entnahmemenge an.

Basis-, Tot- und Greifzeit sind in realen Systemen Zufallsvariablen und es stellt sich die Frage, welcher statistischen Verteilung sie folgen. Viele bedientheoretische Lösungsverfahren basieren auf der Annahme exponentialverteilter Bedienzeiten. Bezogen auf Kommissioniersysteme ist die Zugrundelegung dieses Verteilungstyps jedoch problematisch, da für jede Tätigkeit eine Mindestbearbeitungszeit vorzusehen ist. Da Realisationen nahe Null bei Exponentialverteilungen die höchste Wahrscheinlichkeit haben, ist eine realistische Abbildung der tatsächlichen Bearbeitungszeiten nicht gegeben. Eine besser geeignete Alternative zur Modellierung der Basis-, Tot- und Greifzeiten stellt die logarithmische Normalverteilung dar (Kre-08). Die Zufallsvariable $X$ ist logarithmisch normalverteilt, wenn $\ln (X)$ normalverteilt ist. Eine logarithmisch normalverteilte Zufallsvariable nimmt nur positive Realisationen an. Die rechtsschiefe Form der Verteilung spiegelt die reale Beobachtung wider, dass der Mittelwert der Bearbeitungszeiten rechts vom Modus der Verteilung liegt. In der Realität sind Basis-, Tot- oder Greifzeiten, die größer als die „häufigste“ Basis-, Totoder Greifzeit sind, durchaus möglich, Zeiten darunter sind sehr selten bis nie zu beobachten. Logarithmisch normalverteilte Zeiten lassen sich durch ihren Mittelwert und die Standardabweichung charakterisieren: X LogN(Mittelwert,Standardabweichung). 
Da die Basis-, Tot-, Greif- und Wegzeiten unabhängige Zufallsvariablen bzw. deterministische Variablen sind, lassen sich deren Erwartungswerte sowie Varianzen addieren. Da die Basis-, Tot- und Greifzeit als logarithmisch normalverteilt angenommen werden, lässt sich der Bedienprozess mit Hilfe einer generellen Verteilung mit der mittleren Bedienrate $\mu_{\mathrm{i}}$ und der Variabilität $\mathrm{c}^{2}{ }_{\mathrm{i}}$ charakterisieren. Die Variabilität ist eine Maßzahl für Prozessschwankungen und berechnet sich aus dem Quotienten der Varianz der Bedienzeit und ihrem quadrierten Erwartungswert.

\subsubsection{Modellierung des Routings}

Die Bewegungsstrategie der Kommissionierer legt fest, wie sich diese durch das System bewegen dürfen, d.h. in welcher Reihenfolge die einzelnen Ressourcen (Regalspalten, Stirngänge, Basisstation) besucht werden können. Laut Gudehus (Gud-05) sind in manuellen Kommissioniersystemen mit statischer Artikelbereitstellung folgende Bewegungsstrategien von zentraler Bedeutung:

- Durchlaufstrategie ohne Gangüberspringung

- Durchlaufstrategie mit Gangüberspringung und Einwegverkehr

- Stichgangstrategie mit Gangwiederholung / ohne Gangwiederholung

Bei der Übertragung in ein bedientheoretisches Modell bestimmen die Übergangswahrscheinlichkeiten, wie die einzelnen Bediensysteme miteinander verknüpft werden. Die Übergangswahrscheinlichkeit $\mathrm{q}_{\mathrm{ij}}$ gibt an, mit welcher Wahrscheinlichkeit ein Kunde nach Bearbeitung am Bediensystem i direkt zum Bediensystem $\mathrm{j}$ überwechselt.

\section{Durchlaufstrategie ohne Gangüberspringung}

Bei dieser Strategie durchlaufen die Kommissionierer immer alle Gänge des Kommissioniersystems in einer festen Reihenfolge (siehe Abbildung 4). Dies erfolgt unabhängig davon, ob in einem bestimmten Gang eine Entnahme zu tätigen ist oder nicht. Das entsprechende bedientheoretische Modell besitzt eine rein serielle Anordnung, d.h. jedes Bediensystem i hat genau einen Nachfolger $\mathrm{j}$ und es gilt $\mathrm{q}_{\mathrm{ij}}=1$.

\section{Durchlaufstrategie mit Gangüberspringung und Einwegverkehr}

Sind in einem Gang keine Entnahmen zu tätigen, dürfen bei dieser Strategie Gänge übersprungen werden. Gleichzeitig verhindern Einbahnstraßenregelungen entgegengesetzte Laufwege. Alle Gänge mit gerader Gangzahl dürfen ausschließlich in einer bestimmten Richtung durchlaufen werden, während bei Gängen mit ungerader Gangzahl die entgegengesetzte Richtung vorgeschrieben ist. Komplexe Blockierungen durch Gegenüberstehen (Abb. 2, c) werden so ausgeschlossen.

Zur Übertragung dieser Strategie wird das Bediensystemnetzwerk an verschiedenen Stellen um Verzweigungen erweitert. An diesen muss der Kommissionierer über seinen weiteren Weg entscheiden. Die Verzweigungen liegen jeweils vor dem Ganganfang bzw. nach dem Gangende. Abbildung 5 verdeutlicht diesen Sachverhalt beispielhaft.

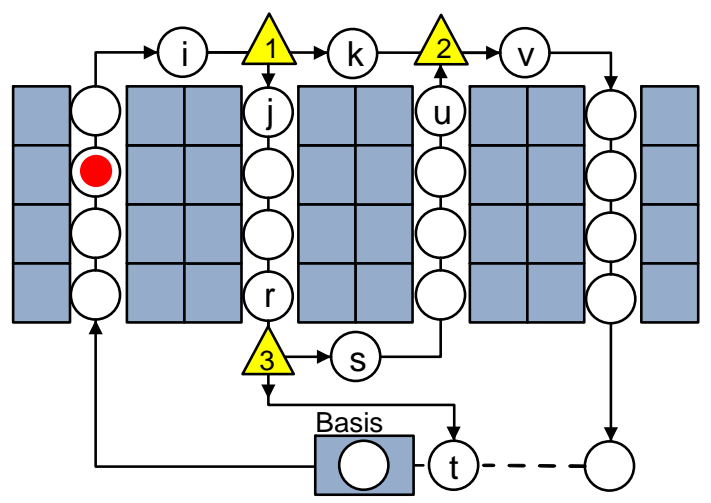

Abbildung 5: Kommissioniersystem als geschlossenes Bediensystemnetzwerk - Durchlaufstrategie mit Gangüberspringung (Einwegverkehr)

Nach Durchlaufen des linken oberen Stirngangs (Bediensystem i) muss der Kommissionierer in Richtung Bediensystem j laufen, wenn er im zweiten oder dritten Gang Entnahmen zu tätigen hat oder zurück zur Basisstation muss (aufgrund der Einbahnstraßenregelung muss der Kommissionierer Gang zwei durchlaufen, auch wenn die nächste Entnahme erst im dritten Gang ist). Findet die nächste Entnahme im vierten Gang statt, 
kann er direkt in Richtung Bediensystem $\mathrm{k}$ und weiter $\mathrm{zu}$ Bediensystem $\mathrm{v}$ gehen. Die Übergangswahrscheinlichkeit $\mathrm{q}_{\mathrm{ij}}$ hängt also von der Wahrscheinlichkeit $\mathrm{ab}$, mit der im zweiten oder dritten Gang eine Entnahme zu tätigen ist und es gilt $\mathrm{q}_{\mathrm{ij}} \in[0 ; 1]$ sowie $\mathrm{q}_{\mathrm{ij}}+\mathrm{q}_{\mathrm{ik}}=1$. Analog dazu entspricht an der dritten Verzweigung $\mathrm{q}_{\mathrm{rs}}$ der Wahrscheinlichkeit, dass der Kommissionierer noch mindestens eine Entnahme in den Gängen drei oder vier durchzuführen hat. $\mathrm{q}_{\mathrm{rt}}$ gibt an, mit welcher Wahrscheinlichkeit der Kommissionierer keine weitere Entnahme vornimmt und zur Basisstation zurückkehrt. Innerhalb der Gänge gibt es für jedes Bediensystem jeweils nur einen Nachfolger. Durch Festlegung einzelner Übergangswahrscheinlichkeiten, z.B. $\mathrm{q}_{\mathrm{uk}}=0$, wird die Einhaltung des Einwegverkehrs garantiert.

Die Höhe der Übergangswahrscheinlichkeiten hängen letztlich von den Entnahmewahrscheinlichkeiten der einzelnen Artikel ab. Werden im obigen Beispiel schnell drehende Artikel ausschließlich im ersten und zweiten Gang, Langsamdreher hingegen in den Gängen drei und vier gelagert, wird $\mathrm{q}_{\mathrm{ij}}>\mathrm{q}_{\mathrm{ik}}$ sein.

\section{Stichgangstrategie mit oder ohne Gangwiederholung}

Bei der Stichgangstrategie bewegen sich die Kommissionierer auf einem Hauptweg, der senkrecht zur Stirnseite der Gänge verläuft. Sofern in einem Gang Entnahmen zu tätigen sind, verlässt der Kommissionierer den Hauptweg. Bewegt sich der Kommissionierer nach jeder Entnahme zurück zum Stirngang - beispielsweise um Waren auf einem Kommissionierwagen abzulegen - und betritt den Gang für eine weitere Entnahme erneut, spricht man von einer Gangwiederholung. Betritt der Kommissionierer den Gang einmalig und tätigt alle Entnahmen, spricht man von einer Stichgangstrategie ohne Gangwiederholung. Sperrt ein Kommissionierer einen Gang bei dessen Betreten für andere Kommissionierer, spricht man von exklusivem Gangrecht. Alternativ ist ein gleichzeitiger Aufenthalt mehrerer Kommissionierer in einem Gang denkbar (nicht-exklusives Gangrecht). Den ersten Fall zeigt Abbildung 6.

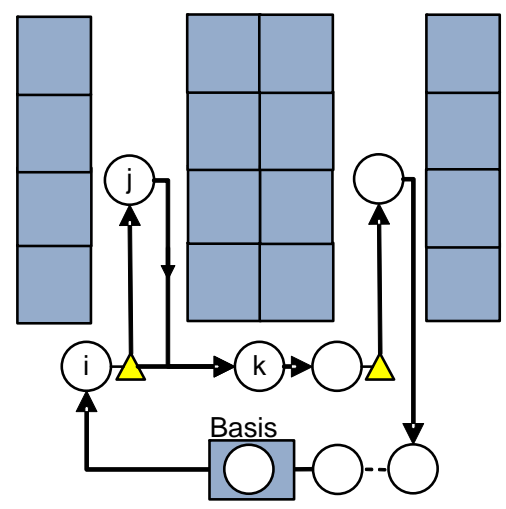

Abbildung 6: Kommissioniersystem als geschlossenes Bediensystemnetzwerk - Stichgangstrategie

Im Unterschied zu den bisherigen Ansätzen wird hierbei nicht jeder Einzelplatz eines Ganges als Bediensystem modelliert. Es erfolgt vielmehr die Modellierung des gesamten Ganges als Einzelplatz mit einer Bedienstation und einem Warteraum der Größe Null. Sobald der erste Kommissionierer den Gang betritt, müssen alle anderen warten. Die Übergangswahrscheinlichkeit $\mathrm{q}_{\mathrm{ij}}$ gibt an, mit welcher Wahrscheinlichkeit der Gang (dargestellt durch Bediensystem j) betreten wird. Die Bedienzeit von $\mathrm{j}$ beinhaltet alle im Gang zu bewältigenden Tot-, Greifund Wegzeiten. Nach Verlassen des Bediensystems j bewegt sich der Kommissionierer zurück auf den Hauptweg, der als Aneinanderreihung einzelner Bediensysteme abgebildet wird. Verfügt der Hauptweg über eine ausreichende Breite, beeinträchtigt eine Warteschlange vor einem Gang die Bewegungen auf dem Hauptweg nicht. Er kann in diesem Fall an den Einmündungsstellen durch zwei parallele Bediensysteme abgebildet werden. Ein Bediensystem bildet dabei die Warteschlange vor dem Gang und das zweite Bediensystem den Hauptweg ab. Die vorgestellte Modellierung ist für eine Stichgangstrategie mit oder ohne Gangwiederholung anwendbar. Bei Gangwiederholungen verlängert sich lediglich die Bedienzeit des (Gang)Bediensystems um die zusätzlichen Wegzeiten. Ist das Gangrecht nicht-exklusiv, kann es zur Problematik des Gegenverkehrs kommen.

In geschlossenen Bediensystemnetzwerken werden die Übergangswahrscheinlichkeiten in so genannte Besuchshäufigkeiten umgewandelt. Die Besuchshäufigkeit $e_{i}$ gibt den relativen Anteil des Gesamtflusses an, der durch das Bediensystem i fließt. Als Zählknoten dient dabei die Basisstation, da diese von den Kommissionierern bei jedem Rundgang besucht werden muss. 
Abbildung 7 fasst die Übertragung der Parameter eines manuellen Kommissioniersystems in die Eingangsgrößen des bedientheoretischen Modells zusammen.

\begin{tabular}{|l|}
\hline Parameter eines Kommissioniersystems \\
\hline Anzahl Kommissionierer \\
\hline Regalspalten, Stirngänge, Basisstation \\
\hline Basis-, Tot-, Greif- und Wegzeiten \\
\hline Auftragsspezifische Daten \\
\hline Bewegungsstrategien \\
\hline Artikelspezifische Daten \\
\hline
\end{tabular}

\begin{tabular}{|c|}
\hline Bedientheoretische Modellierung \\
\hline Anzahl Kunden im System k \\
\hline $\begin{array}{l}\text { Anzahl Bediensysteme } n \\
\text { Kapazitäten der Bediensysteme } m_{i}\end{array}$ \\
\hline Bedienrate $\mu_{\mathrm{i}}$, Variabilität $\mathrm{c}^{2}{ }_{\mathrm{i}}$ \\
\hline Bedienrate $\mu_{\mathrm{i}}$, Variabilität $\mathrm{c}^{2}{ }_{\mathrm{i}}$, Besuchshäufigkeiten $\mathrm{e}_{\mathrm{i}}$ \\
\hline Besuchshäufigkeiten $\mathbf{e}_{\mathbf{i}}$ \\
\hline Besuchshäufigkeiten $\mathbf{e}_{\mathbf{i}}$ \\
\hline
\end{tabular}

Abbildung 7: Parameter eines Kommissioniersystems und deren bedientheoretische Modellierung

\subsection{Kennwertberechnung für das bedientheoretische Modell}

Der Übertragung eines manuellen Kommissioniersystems in ein bedientheoretisches Modell liegen folgende Annahmen zugrunde:

- Geschlossenes Bediensystemnetzwerk mit n Bediensystemen und k Kunden

- Wahlweise serielle oder verzweigte Anordnung der einzelnen Bediensysteme

- Kapazitäten der Bediensysteme $\mathrm{m}_{\mathrm{i}}$ sind jeweils 1, d.h. die Größe des Warteraums ist Null

- Bedienzeiten folgen einer generellen Verteilung (mittlere Bedienrate $\mu_{\mathrm{i}}$ und Variabilität $\mathrm{c}^{2}{ }_{\mathrm{i}}$ )

Aufgrund dieser Anforderungen sind zahlreiche Berechnungsverfahren der Bedientheorie nicht anwendbar. Besonders die Annahme nicht-exponentialverteilter Bedienzeiten sowie die beschränkte Größe des Warteraums verkleinern den Kreis potentieller Verfahren enorm.

In der Literatur konnten die Verfahren von Akyildiz aus dem Jahre 1987 (Aky-87), Bouhchouch et al. (Bou-96) sowie Rall (Rall-98) als mögliche Lösungsansätze identifiziert werden. Akyildiz zeigt in seiner Veröffentlichung (Aky-87) jedoch große relative Fehler von 20-25\% auf. Der Ansatz von Bouhchouch et al. wird der Klasse der Dekompositionsverfahren zugeordnet und tendiert für größere Netzwerke zu einem hohen Rechenaufwand. Aufgrund der genauen Ergebnisse, die besonders für zyklische Netzwerke berichtet wurden, erscheint die Methode von Rall als geeigneter Lösungsansatz.

Rall kombiniert zahlreiche etablierte Lösungsverfahren zu einem neuen Berechnungsansatz. Dabei greift er im ersten Schritt auf Teile einer im Jahr 1988 von Akyildiz (Aky-88) entwickelten Methode zurück. Akyildiz transformiert ein Blockiernetz (Bediensystemnetzwerk mit beschränkten Warteräumen) mit exponentialverteilten Bedienzeiten und $\mathrm{k}$ Kunden in ein äquivalentes Nicht-Blockiernetz mit neuer Kundenanzahl $\mathrm{k}^{*}$, wobei ${ }^{*} \leq \mathrm{k}$ gilt. Aufgrund der Annahme exponentialverteilter Bedienzeiten vernachlässigt Akyildizs Ansatz jedoch den Einfluss von Variabilitäten $\mathrm{c}^{2}{ }_{\mathrm{i}} \neq 1$.

Die Methode von Rall passt die reduzierte Kundenanzahl $\mathrm{k}^{*}$ mit Hilfe eines Korrekturfaktors $\mathrm{f}$ gemäß $\mathrm{k}^{* *}=\mathrm{k}^{*} \cdot \mathrm{f}$ an. Der Korrekturfaktor f hängt u.a. von der Variabilität der einzelnen Bedienstationen ab. Ebenso hat das Verhältnis zwischen den aktuell im System befindlichen Kunden sowie der maximal möglichen Anzahl im System befindlicher Kunden Einfluss. Zur Berechnung von $\mathrm{f}$ greift Rall auf die Mittelwertanalyse als wichtiges Verfahren zur Bestimmung des Durchsatzes bei unendlich großen Warteräumen und exponentialverteilten Bedienzeiten (Bol-98) zurück.

Im letzten Schritt wendet Rall die Methode von Marie (Mar-80) mit der Kundenanzahl k** an. Das iterative Verfahren berechnet zunächst für jedes Bediensystem die Ankunftsraten in Abhängigkeit der Kundenanzahl. Je nach Höhe der Variabilität werden die Bedienprozesse anschließend durch mehrphasige Cox-Verteilungen modelliert und die Bedienraten in Abhängigkeit der Kundenanzahl bestimmt. Maries Ansatz dient ursprünglich der Lösung von geschlossenen Bediensystemnetzwerken mit unendlich großen Warteräumen und generellen Bedienzeiten. Durch die Nutzung der angepassten Kundenanzahl $\mathrm{k}^{* *}$ ermöglicht Rall jedoch die Berücksichtigung des Einflusses beschränkter Warteräume. 


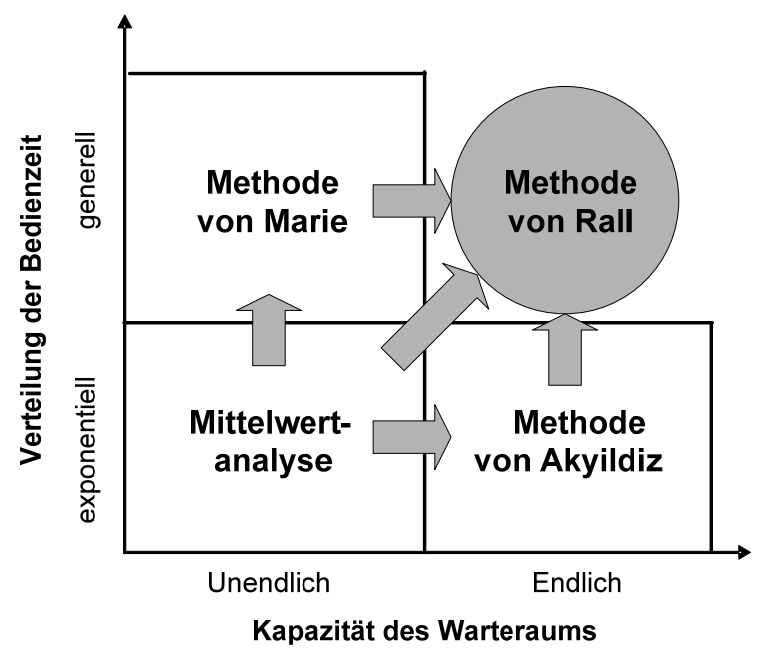

Abbildung 8: Ausgewählte Lösungsverfahren für geschlossene Bediensystemnetzwerke

\section{Beispielberechnung von Leistungskennwerten für ein manuelles Kommissioniersystem}

\subsection{Modellierung eines Beispielsystems}

Die Berechnung von Leistungskennwerten dient der Bestimmung der Ergebnisgüte der entwickelten bedientheoretischen Modellierung. Zunächst ist dabei die Konzeption eines repräsentativen manuellen Kommissioniersystems erforderlich. Darauf basierend werden Leistungskennwerte anhand eines bedientheoretischen Modells und mittels Simulation berechnet sowie gegenübergestellt.

Es wird angenommen, dass das Beispielsystem eine Basisstation besitzt. Der Aufbau entspricht einem Blocklayout mit vier Gängen zu je 15 Einzelplätzen (= 30 Regalspalten). Die Länge eines Ganges sei 15m. Eine Regalspalte sei 0,75m tief, die Gesamtlänge eines Stirngangs 3m. Der Weg vom letzten Gang zurück zur Basis sei $5 \mathrm{~m}$. Gänge, Stirngänge und die Basis seien jeweils ein Einzelplatz, während der Weg zur Basis aus fünf Einzelplätzen besteht. Somit können fünf Kommissionierer vor der Basis warten, ohne Blockiervorgänge auszulösen. Es existieren insgesamt 69 Einzelplätze. Pro Auftrag sind 10 Entnahmen zu tätigen. Die Entnahmewahrscheinlichkeit ist für alle Regalfächer gleich. An der Basis liegen stets Aufträge zur Bearbeitung bereit. Die Geschwindigkeit der Kommissionierer sei konstant $1 \mathrm{~m} / \mathrm{s}$, Beschleunigungen werden vernachlässigt. Die Kommissionierer bewegen sich unter Anwendung der Durchlaufstrategie ohne Gangüberspringung durch das System. Tot-, Greif- und Basiszeit seien logarithmisch normalverteilt und variieren in den verschiedenen Untersuchungsszenarien. Für alle Szenarien wird angenommen, dass Tot- und Greifzeit in einer gemeinsamen Zufallsvariable abgebildet werden.

Unter Berücksichtigung dieser Annahmen erfolgt die Übertragung des Beispielsystems in ein bedientheoretisches Modell (Abbildung 9).
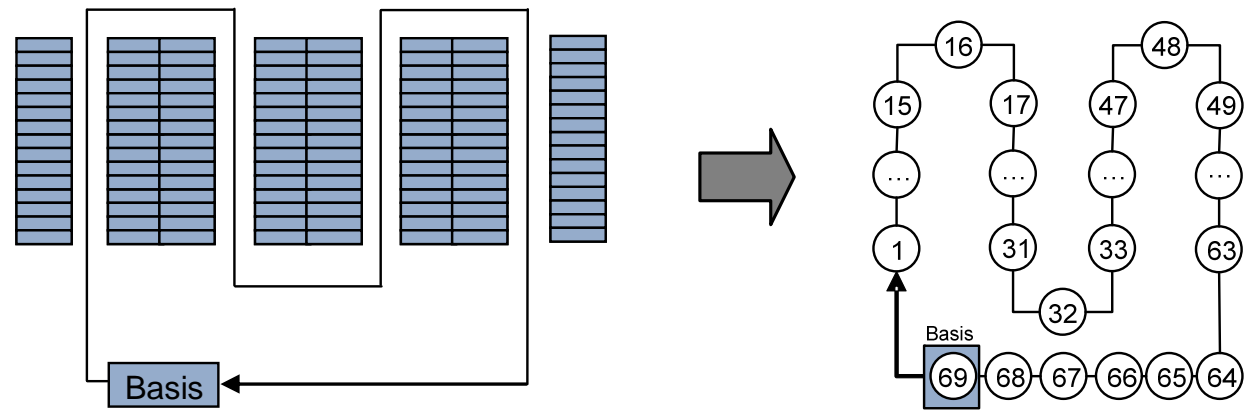

Abbildung 9: Beispielsystem als bedientheoretisches Modell

Gemäß der eingeführten Modellierungslogik wird jeder Einzelplatz des Beispielsystems als Bediensystem mit generell verteilter Bedienzeit abgebildet. Die Bediensysteme 16, 32 und 48 stellen die Stirngänge dar, während die Bediensysteme 64-68 den Weg vom letzten Gang zur Basisstation abbilden. Bediensystem 69 repräsentiert 
die Basisstation. Die verbleibenden Bediensysteme modellieren Einzelplätze in den Gängen. Die Systemkapazitäten $\mathrm{m}_{\mathrm{i}}$ sind jeweils 1, gleiches gilt aufgrund der Laufstrategie der Kommissionierer für die Besuchhäufigkeiten $\mathrm{e}_{\mathrm{i}}$.

\subsection{Ergebnisvergleich: Simulation - Bedientheorie - Statisches Durchsatzmodell}

Zur Quantifizierung der Durchsatzverluste wird das Beispielsystem zunächst in einem Simulationsmodell abgebildet. Die Abläufe und dynamischen Effekte im Beispielsystem werden in der Simulation detailliert wiedergegeben. Aus diesem Grund kann angenommen werden, dass die Ergebnisse der Simulation dem „realen Durchsatzverlauf des Beispielsystems“ entsprechen und somit Referenzwerte darstellen. Der Vergleich mit den berechneten Kennwerten des bedientheoretischen Modells zeigt, dass die Bedientheorie geeignet ist, die dynamischen Effekte in manuellen Kommissioniersystemen zu berücksichtigen. Zusätzlich werden die Referenzwerte den Ergebnissen eines statischen Durchsatzmodells, das keine Blockiervorgänge abbildet, gegenübergestellt. So zeigt sich, inwiefern ein statisches Berechnungsmodell reale Durchsätze überschätzt.

Für die Tot- und Greifzeit gelte $t_{\text {Tot }+ \text { Greif }} \sim \operatorname{LogN}(15,10)$. Für Bediensysteme, die einen Einzelplatz im Gang darstellen, muss die Bedienzeit sowohl den Fall der Entnahme, als auch das Passieren der Regalspalte berücksichtigen. Mit Hilfe der Entnahmewahrscheinlichkeit $p_{i}=1 / 6$ (10 Entnahmen pro Auftrag bei 60 möglichen Entnahmeplätzen) wird die mittlere Bedienzeit berechnet. Für die $\operatorname{LogN}(15,10)$-verteilte Tot- und Greifzeit und eine Laufzeit von 1s ergibt sich eine mittlere Bedienzeit von 3,5 Sekunden $(=1 / 6 \bullet(15+1)+5 / 6 \bullet$ (0+1). Zur Bestimmung der Variabilität werden Zufallszahlen im Verhältnis 1/6 (gemäß $\operatorname{LogN}(15,10)$ ) zu 5/6 (konstante Laufzeit von 1Sek mit Varianz $=0$ ) erzeugt und die benötigten statistischen Kennwerte abgeleitet. Die Basiszeit sei verteilt gemäß $t_{\text {Basis }} \sim \operatorname{LogN}(10,5)$. Die Bedienzeiten der Stirngänge und Rückwege seien deterministisch 1 Sek. Die Kennwerte des bedientheoretischen Modells werden mit dem Verfahren von Rall berechnet.

Um den Auftragsdurchsatz mit Hilfe eines statischen Berechnungsmodells zu bestimmen, wird das klassische Wegzeitmodell von Gudehus (Gud-05) herangezogen. Mit den Annahmen des Beispielsystems berechnet sich die mittlere Anzahl Gänge x, in denen die 10 Entnahmen bei Gleichverteilung zu finden sind, zu 3,775. Die mittlere Weglänge pro Auftrag L(4 Gänge) ist dann 67,115m und mit der gegebenen Geschwindigkeit ergibt sich eine Wegzeit von 67,115 Sekunden pro Auftrag. Zuzüglich der gesamten Tot- und Greifzeit von 150 Sekunden und der Basiszeit von 10 Sekunden resultiert eine Gesamtauftragsdurchlaufzeit von ca. 227 Sekunden und der Auftragsdurchsatz pro Stunde ist schließlich 15,85.

Unter der Annahme, dass der Zusammenhang zwischen der Anzahl Kommissionierer und dem Auftragsdurchsatz im statischen Ansatz linear ist, zeigt Abbildung 10 die Durchsatzverläufe der drei unterschiedlichen Berechnungsmethoden.

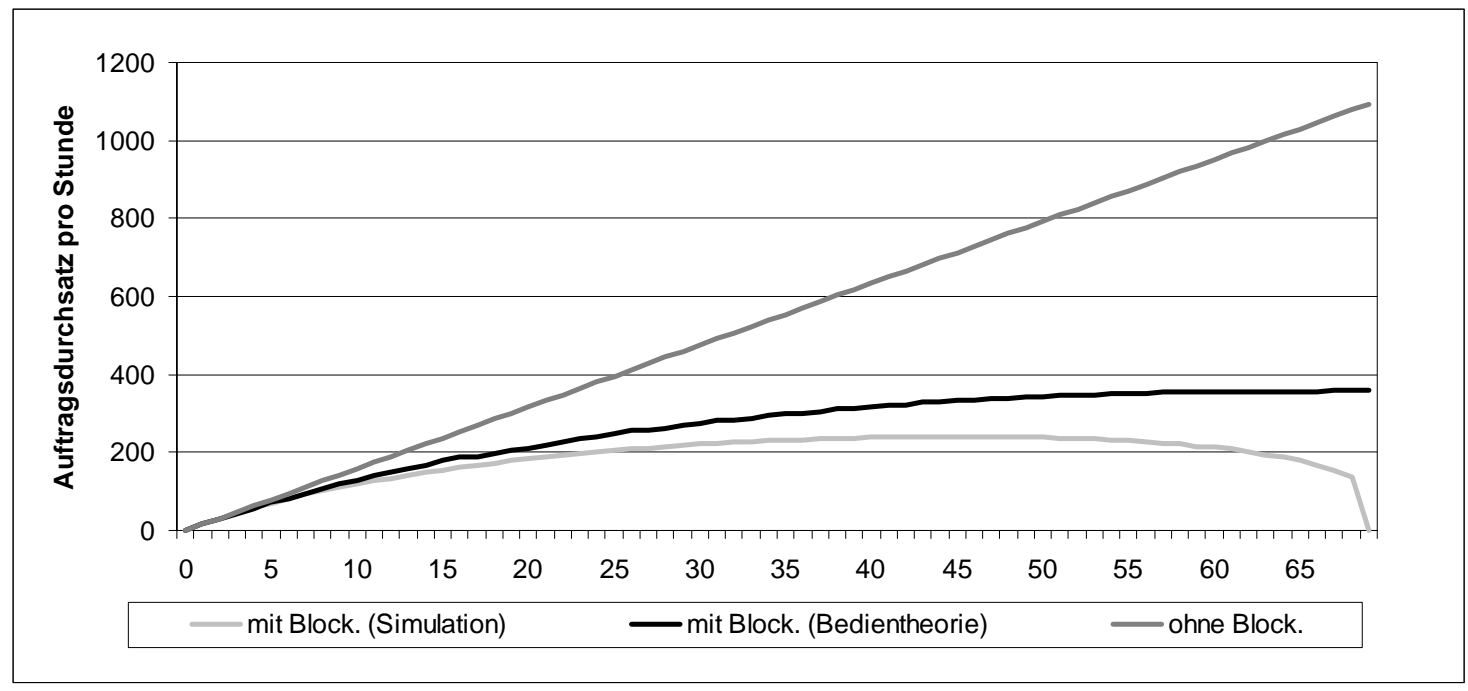

Abbildung 10: Durchsatzverläufe für verschiedene Berechnungsmethoden

Es zeigt sich, dass der Durchsatz ohne Berücksichtigung von Blockiervorgängen konstant zunimmt. Die mittels Bedientheorie berechneten Durchsatzwerte hingegen beinhalten die dynamischen Effekte und nähern sich für 
wachsende Anzahl Kommissionierer einer Sättigungsgrenze an. Der Verlauf der simulativ berechneten Durchsätze zeigt ebenfalls die dynamischen Effekte und spiegelt darüber hinaus die intuitive Vermutung wider, dass ab einer bestimmten Anzahl Kommissionierer die Blockiervorgänge den zusätzlichen Grenzdurchsatz eines weiteren Kommissionierers überkompensieren. Der fallende Verlauf der Durchsatzkurve endet letztlich in einer Deadlock-Situation und der Auftragsdurchsatz beträgt 0. Im mittleren und rechten Bereich des Diagramms wird die Abweichung zwischen Bedientheorie und Simulation zunehmend größer. Ein Grund hierfür ist in den Lösungsverfahren zu sehen, auf die das Verfahren von Rall zurückgreift (Abbildung 8). Balsamo, de Nitto Personé und Onvural (Bal-01) merken an, dass der Fehler im Verfahren von Akyildiz (Aky-88) wächst, je größer die untersuchten Netzwerke sind. Eine Verbesserung der Verfahren muss daher Inhalt der weiteren Forschungsarbeiten sein. Aus Sicht der praktischen Anwendung ist der mittlere und rechte Bereich des obigen Diagramms zu vernachlässigen, da reale Kommissioniersysteme meist mit einer begrenzten Anzahl an Mitarbeitern ( $<<$ Anzahl Einzelplätze) betrieben werden.

Die Überschätzung des realen Durchsatzes in Abhängigkeit der gewählten Berechnungsmethode ergibt sich mit Hilfe der folgenden Formeln:

$$
\begin{gathered}
\Delta=\left(\mathrm{X}_{\text {Statisches Modell }}-\mathrm{X}_{\text {Simulation }}\right) / \mathrm{X}_{\text {Simulation }} \\
\Delta=\left(\mathrm{X}_{\text {Bedientheorie }}-\mathrm{X}_{\text {Simulation }}\right) / \mathrm{X}_{\text {Simulation }}
\end{gathered}
$$

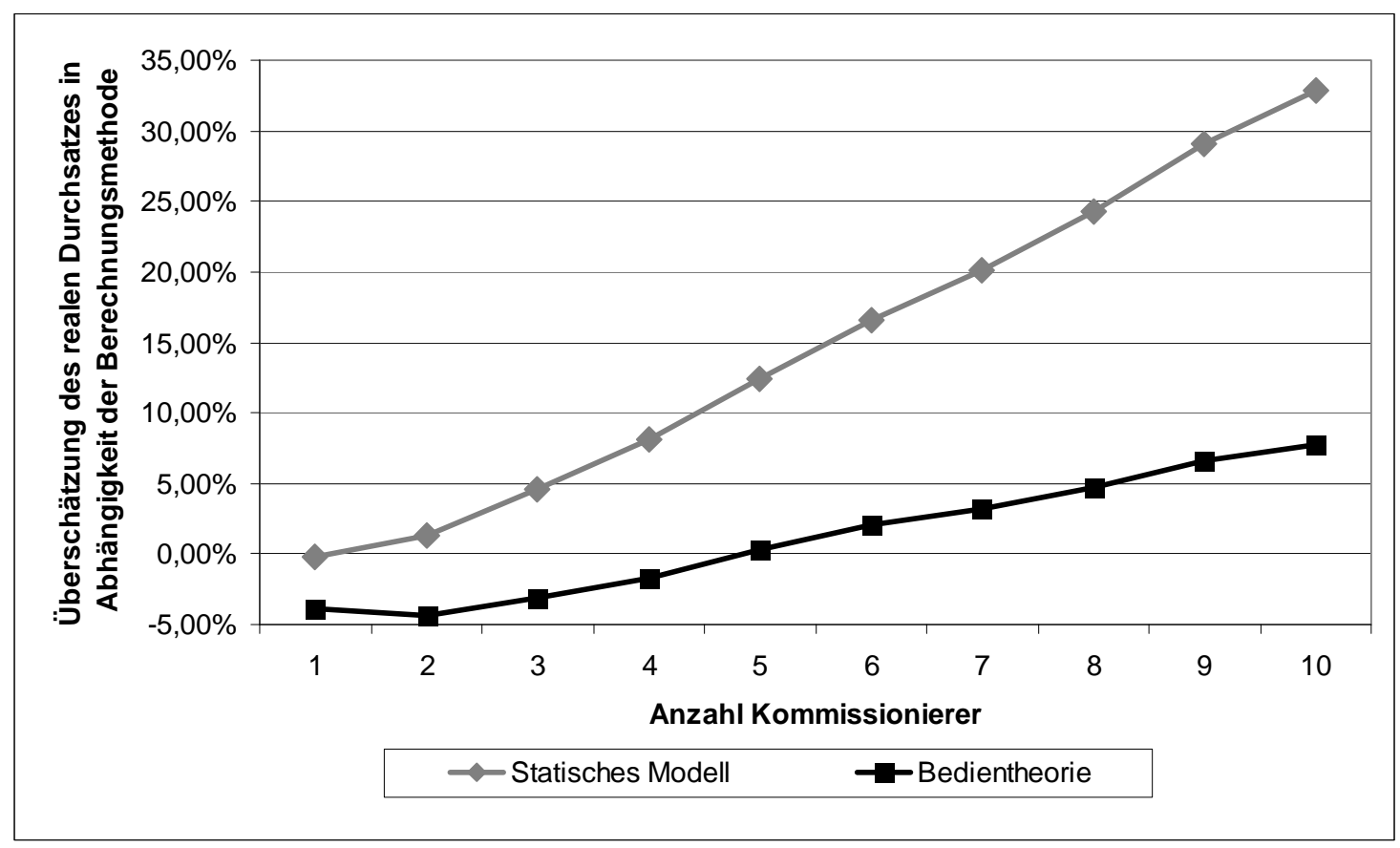

Abbildung 11: Überschätzung des realen Durchsatzes

Abbildung 11 verdeutlicht die Abweichungen zwischen den Ergebnissen der Simulation und jeweils den Ergebnissen des statischen bzw. bedientheoretischen Modells. Die Ergebnisse des bedientheoretischen Modells liegen im akzeptablen Bereich von $+/-5 \%$, sofern weniger als 9 Kommissionierer im System arbeiten (so dass $12 \%$ der Einzelplätze im System maximal belegt sind). Eine Anwendung statischer Berechnungsmodelle ohne Berücksichtigung von Blockiervorgängen führt hingegen schnell zu einer deutlichen Überschätzung des Durchsatzes.

Neben der Durchsatzleistung lässt sich das System auch aus Kostensicht bewerten. Dazu wird beispielsweise die Produktivitätskennzahl „Anzahl der Entnahmeposition pro Mitarbeiterstunde“ bestimmt. Daraus lässt sich ableiten, wie hoch die Kosten pro Entnahmeposition sind. Abbildung 12 zeigt, dass die Produktivität im Falle der statischen Berechnungsmethode auf konstantem Niveau bleibt. Im Gegensatz zeigen sowohl die Simulation als auch das bedientheoretische Modell die Produktivitätsverluste auf.

Die Vernachlässigung von Blockiervorgängen durch ein statisches Berechnungsmodell resultiert in der Grobplanungsphase manueller Kommissioniersysteme letztlich in einer falschen Einschätzung der Systemleistung und somit in einer fehlerhaften Auslegung des Systems. Darüber hinaus werden die Kosten 
unterschätzt, da die einzelne Entnahmeposition letztlich mit zu geringen Kosten bewertet wird. Die Ergebnisse des bedientheoretischen Modells hingegen folgen dem realen Durchsatzverlauf und erlauben eine realistischere Einschätzung der zu erwartenden Systemleistung bzw. der zu erwartenden Kosten.

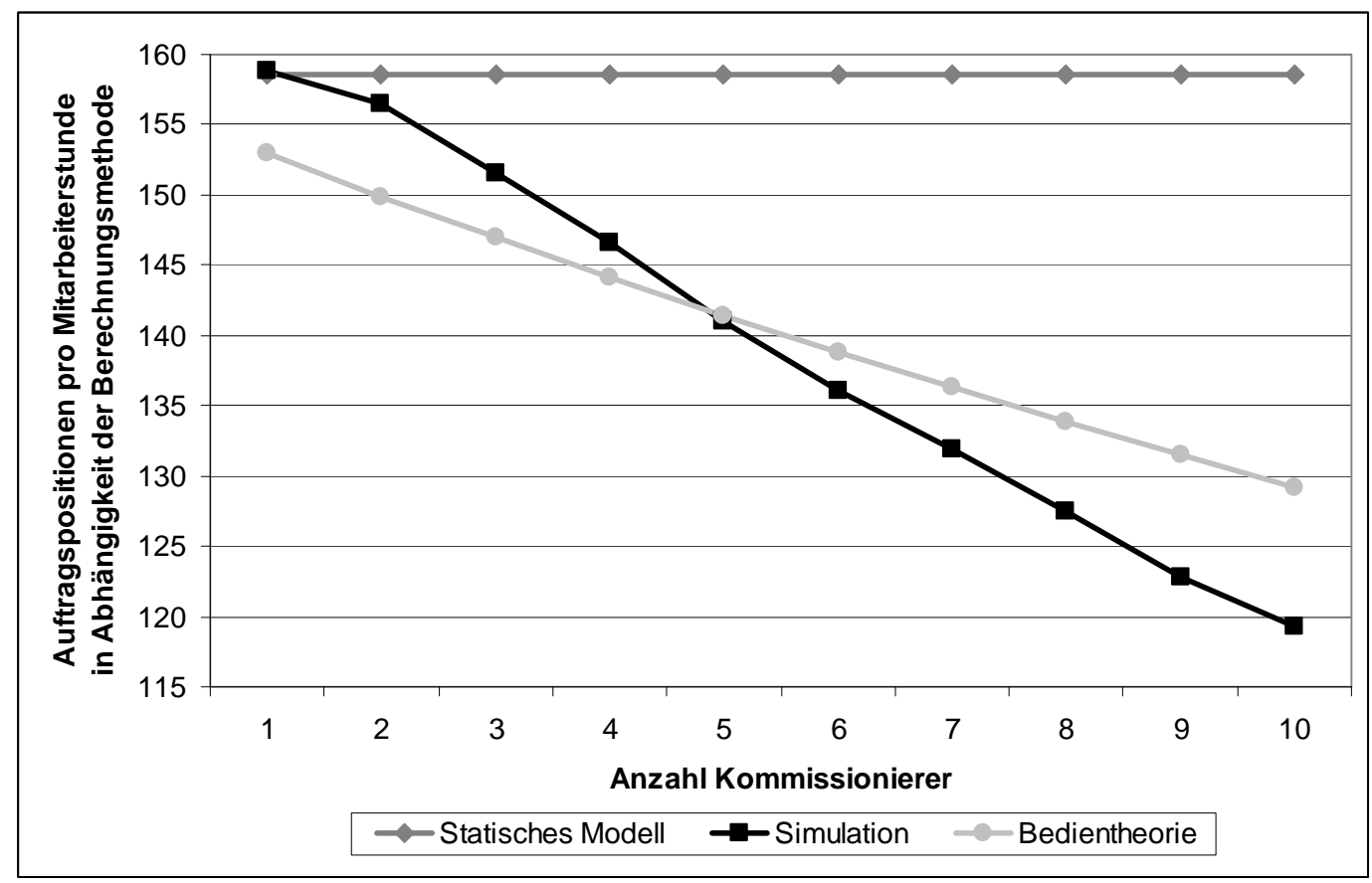

Abbildung 12: Produktivitätsverluste aufgrund von Blockiervorgängen

\subsection{Ergebnisvergleich: Simulation - Bedientheorie}

Im Folgenden werden die Referenzwerte der Simulation mit den Kennwerten des bedientheoretischen Modells im praxisrelevanten Bereich detaillierter gegenübergestellt. Im ersten Szenario gelte für die Tot- und Greifzeit $\mathrm{t}_{\text {Tot+Greif }} \sim \operatorname{LogN}(15,10)$. Die Standardabweichung der Basiszeit beträgt 5 Sek. Die mittlere Basiszeit variiert zwischen den Werten 10, 20 und 30. Die Herleitung der Bedienzeiten und Variabilitäten erfolgt analog zu Kapitel 5.2 anhand der Entnahmewahrscheinlichkeiten $p_{i}=1 / 6$. Wiederum dient das Verfahren von Rall zur Kennwertberechnung. Abbildung 13 zeigt die prozentualen Abweichungen der Ergebnisse in einem ,praxisrelevanten Bereich“.

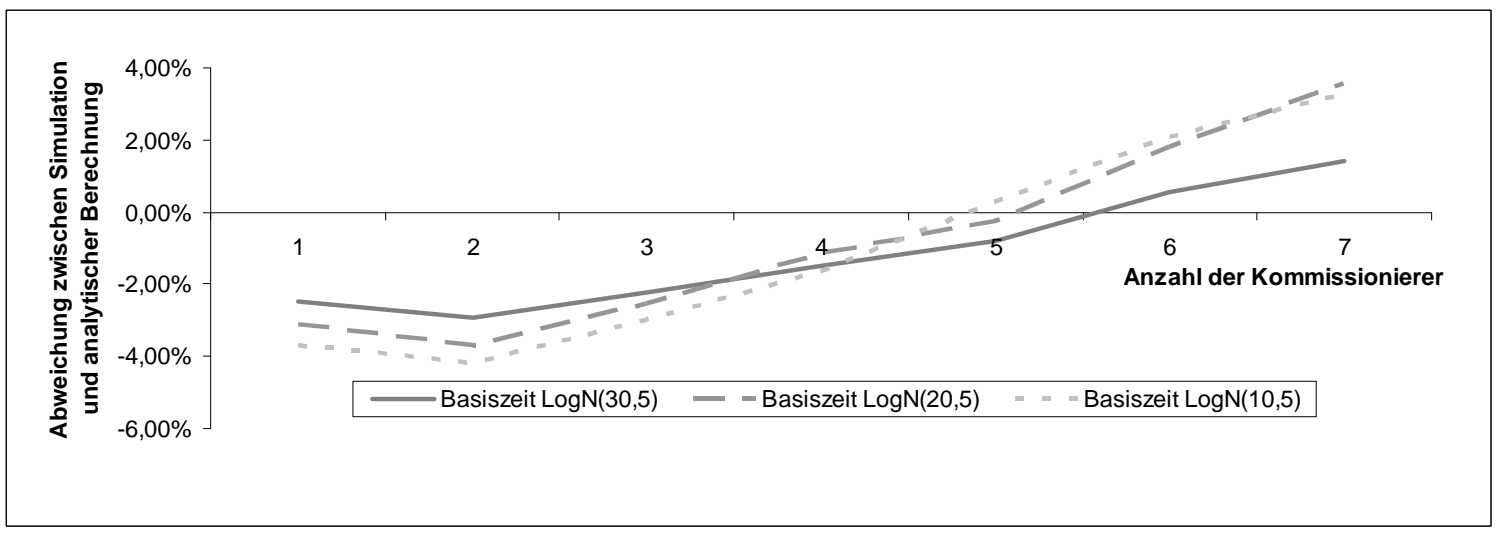

Abbildung 13: Prozentuale Abweichungen zwischen Simulation und bedientheoretischer Berechnung bei fixer Tot- und Greifzeit

Zunächst liegt der Auftragsdurchsatz der Simulation über dem der analytischen Berechnung. Ab einer bestimmten Anzahl Kommissionierer liefert die bedientheoretische Modellierung jedoch höhere Durchsatzwerte. Bei einer mittleren Basiszeit von 30 Sek. ist die relative Abweichung zu vernachlässigen. Dies gilt auch bei einer Reduktion der mittleren Basiszeit auf 20 bzw. 10 Sek., sofern die Anzahl der Kommissionierer gering ist. Wird diese Anzahl größer, steigt auch die Abweichung und liegt zum Beispiel für 10 Kommissionierer bei ca. 8\%. 
Die Abweichungen werden größer, wenn die Basisstation keinen Engpass darstellt und die Variabilitäten an den Entnahmeplätzen steigen (vgl. Abbildung 14). Bei einer mittleren Basiszeit von 30 Sekunden kann die Basisstation bereits für eine geringe Anzahl an Kommissionierern als Engpass identifiziert werden. Die Parameter der Basisstation werden dann zum entscheidenden Einflussfaktor des Durchsatzes. Sinkt die mittlere Basiszeit, verringert sich auch der Engpasseffekt an der Basisstation. Die Bedienstationen im Gang agieren dann als Durchsatztreiber und ihre Variabilitäten beeinflussen das Ergebnis der bedientheoretischen Berechnung. Die Abweichungen werden größer, je höher die Variabilitäten an den einzelnen Entnahmeplätzen sind. Da die Entnahmewahrscheinlichkeit lediglich 1/6 beträgt, ist zumeist das Passieren (Bedienzeit = 1sek) der Einzelplätze im Gang zu beobachten. Für $\mathrm{t}_{\text {Tot+Greif }} \sim \operatorname{LogN}(15,10)$ ergibt sich insgesamt eine Variabilität von 3,8837.

In einem zweiten Szenario wird die Standardabweichung der Tot- und Greifzeit variiert und die Auswirkungen untersucht. An den einzelnen Bediensystemen im Gang gelte stets eine mittlere Bedienzeit von 15 Sek. Die zugehörige Standardabweichung variiert zwischen den Werten 5, 20 und 25 Sek. Die Basiszeit sei LogN(10,5)verteilt. Die Abweichungen der mittels Bedientheorie und Simulation berechneten Durchsätze vergrößern sich bei hohen Variabilitäten (siehe Abbildung 14). Für große Standardabweichungen besitzt der Bedienprozess an den Entnahmeplätzen sehr hohe Variabilitäten von 7,35 $\left(\mathrm{t}_{\text {Tot }+ \text { Greif }} \sim \operatorname{LogN}(15,20)\right)$ bzW. 10,57 $\left(\mathrm{t}_{\text {Tot }+ \text { Greif }} \sim\right.$ $\operatorname{LogN}(15,25))$. Im Fall der LN(15;7,5)-verteilten Tot- und Greifzeit ist die Variabilität vergleichsweise gering. Die Abweichungen bewegen sich im ,praxisrelevanten Bereich“ für diesen Fall innerhalb von 5\%.

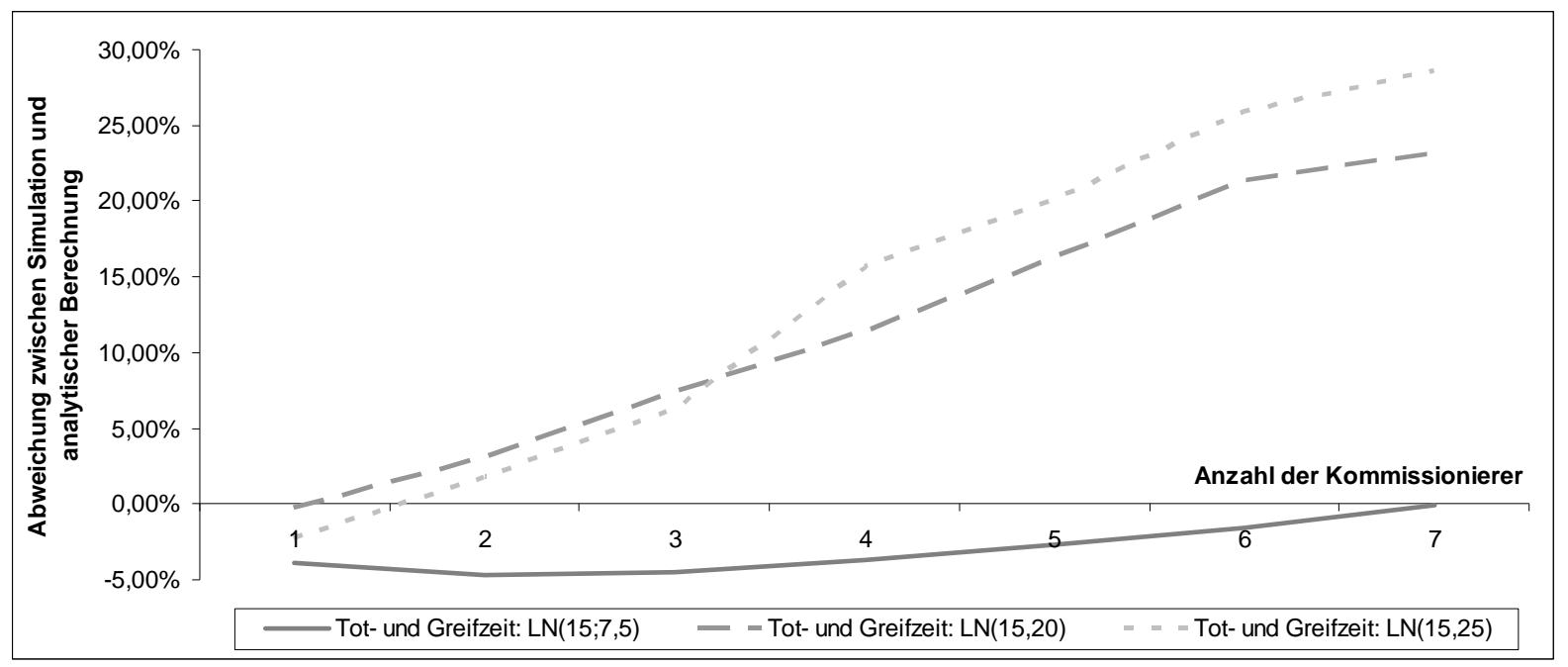

Abbildung 14: Prozentuale Abweichungen zwischen Simulation und analytischer Berechnung - fixe Basiszeit

Die untersuchten Szenarien zeigen, dass analytisch berechnete Kennwerte in Bezug auf Simulationsergebnisse bei geringen Variabilitäten der Tot- und Greifzeit eine gute Abbildungsgüte erreichen. In den gezeigten Beispielen macht die Basiszeit ca. 5-12\% der theoretischen Auftragsdurchlaufzeit aus. Gudehus (Gud-05) beziffert den Anteil der Basiszeit auf ca. 10\%, so dass die Konformität mit Beispielen aus der Literatur gewährleistet ist. Für Variabilitäten $\mathrm{c}^{2}{ }_{\text {Tot+Greifzeit }}>1$ werden die Abweichungen zwischen Simulationsergebnissen und analytischer Berechnung zunehmend größer. Diese liegen im Bereich von drei oder weniger Kommissionierern aber noch unterhalb von 10\%. Ein weiterer Ansatzpunkt für zukünftige Forschungsarbeiten ist eine tiefer gehende Analyse der Auswirkungen großer Variabilitäten und Möglichkeiten, diese in den bestehenden Verfahren besser zu berücksichtigen.

Die Bedientheorie bietet gegenüber der Simulation allgemein den Vorteil einer schnellen Modellierung und Ergebniserzeugung. Die vorgestellten Konzepte zur bedientheoretischen Modellierung von manuellen Kommissioniersystemen unter Berücksichtigung von Blockiervorgängen lassen sich einfach auf zahlreiche Systeme anwenden. Eine schnelle Berechnung von Kennwerten wird durch die präsentierten Lösungsverfahren ermöglicht. 


\section{Zusammenfassung und Ausblick}

In Kommissioniersystemen, dem größten Kostenfaktor intralogistischer Systeme, treten Effizienzverluste bereits bei geringer Anzahl an Kommissionierern auf, da diese sich in den Gängen bzw. an der Basisstation blockieren. Im Rahmen des von der Deutschen Forschungsgemeinschaft (DFG) geförderten Forschungsvorhabens „Blockiervorgänge in manuellen Kommissioniersystemen mit statischer Artikelbereitstellung“ sollen theoretische Grundlagen zur Beschreibung und Quantifizierung von Blockiervorgängen entwickelt werden.

Für die Analyse von Systemen mit stochastischen Einflüssen wurde die Bedientheorie als leistungsfähiges Werkzeug identifiziert. Mit Hilfe der vorgestellten Modellierungsansätze lassen sich manuelle Kommissioniersysteme - bestehend aus den Hauptkomponenten Basisstation, Gang und Stirngang - Schritt für Schritt in geschlossene Bediensystemnetzwerke übertragen. Charakteristische Eigenschaften der Kommissioniersysteme, wie zum Beispiel die Wegstrategie, lassen sich mit Hilfe verschiedener Parameter im bedientheoretischen Modell berücksichtigen. Die Methode von Rall ist auf geschlossene Bediensystemnetzwerke mit beschränkten Warteräumen und generell verteilten Bedienzeiten anwendbar. Sie erfüllt damit die aus der Modellierung resultierenden Anforderungen an ein Lösungsverfahren.

Folgende Erkenntnisse aus Simulation und Anwendung des bedientheoretischen Modells sind hervorzuheben:

- In manuellen Kommissioniersystemen führen Blockierungen bereits bei einer geringen Anzahl Kommissionierer zu Durchsatzverlusten

- Die Produktivität der Mitarbeiter, gemessen anhand der Anzahl Entnahmepositionen pro Mitarbeiterstunde, verringert sich mit jedem zusätzlich eingesetzten Kommissionierer spürbar

- Im Gegensatz zu statischen Berechnungsansätzen sind sowohl Simulation als auch Bedientheorie in der Lage, den aus Blockiervorgängen resultierenden Durchsatzverlust zu quantifizieren

- Die Bedientheorie bietet sich als Modellierungsmethode an, da sie im Vergleich zur Simulation mit sehr geringem Zeitaufwand Ergebnisse vergleichbarer Güte berechnen kann

- Die relative Abweichung zwischen Simulationsmodell und analytischer Berechnung liegt bei geringen Variabilitäten unter 5\%, wird aber mit steigender Variabilität größer. 


\section{Literatur}

[Arn-03]

[Aky-87]

[Aky-88]

[Bal-01]

[Bol-98]

[Bou-96]

[DCRM-08]

[Fai-02]

[Gud-05]

[Gue-05]

[Hall-93]

[Kun-75]

[Kre-08]

[Lün-05]

[Mar-80]

[Rall-98]

[Rat-83]

[Rood-01]

[Rub-99]
Arnold, D.: Abschlussbericht Modellierung und Optimierung von mehrstufigen

Kommissioniersystemen (Forschungsvorhaben AR 160/14-1 der Deutschen

Forschungsgemeinschaft), 2003.

Akyildiz, I.F.: General Closed Queueing Networks with Blocking. In: Proceedings of the 12th IFIP WG 7.3 International Symposium on Computer Performance Modelling, Measurement and Evaluation. Amsterdam: North-Holland 1987.

Akyildiz, I.F.: On the Exact and Approximate Throughput Analysis of Closed Queueing Networks with Blocking. IEEE Transactions on Software Engineering 14(1), 1988, S. 62-70. Balsamo, S., de Nitto Personé, V. und Onvural, R.: Analysis of queueing networks with blocking. Norwell: Kluwer Academic Publishers 2001.

Bolch, G., Greiner, S., de Meer, H., Trivedi, K.S.: Queueing networks and Markov chains: modeling and performance evaluation with computer science applications. New York: John Wiley \& Sons Inc. 1998.

Bouhchouch, A., Frein, Y. und Dallery, Y.: Performance Evaluation of closed tandem queueing networks with finite buffers. Performance Evaluation 26, 1996, S. 115-132

Distribution Center Reference Model (DCRM): Ergebnisse der fortlaufenden Studie „Warehouse Excellence“, Institut für Fördertechnik und Logistiksysteme, Universität Karlsruhe (TH), 2008.

Faißt, B., Lippolt, C. : Staueffekte in Materialflusssystemen. F+H Fördern und Heben 52(8), 2002, S.506-508.

Gudehus, T.: Logistik (Grundlagen - Strategien - Anwendungen). Berlin: Springer 2005.

Gue, K.R., Meller, R.D. und Skufca, J.D.: The effects of pick density on order picking areas with narrow aisles. IEE Transactions 38, 2005, S. 859-868.

Hall, R.W.: Distance approximations for routing manual pickers in a warehouse. IIE

Transactions 25(4), 1993, S. 76-87.

Kunder, R. und Gudehus, T.: Mittlere Wegzeiten beim eindimensionalen Kommissionierern.

Zeitschrift für Operations Research 19, 1975, S. B53-B72.

Krengel, M., Turek, K.: Modellierung der Dynamik manueller Operationen in logistischen Systemen. 4. Fachkolloquium der Wissenschaftlichen Gesellschaft für Technische Logistik, Dresden, 2008.

Lüning, R.: Beitrag zur optimierten Gestaltung des Durchsatzes in Kommissioniersysteme für Stückgüter. Göttingen: Cuvillier 2005, zugl. Dissertation Technische Universität Ilmenau.

Marie, R. : Calculating Equilibrium Probabilities for $\lambda(\mathrm{n}) / \mathrm{C}_{\mathrm{k}} / 1 / \mathrm{N}$ Queues. ACM Computing Surveys 9(2), S. 117-125.

Rall, B.: Analyse und Dimensionierung von Materialflusssystemen mittels geschlossener Warteschlangennetze. Dissertation. Wissenschaftliche Berichte des Institutes für Fördertechnik und Logistiksysteme der Universität Karlsruhe (TH), Band 46, 1998.

Ratliff, H.D. und Rosenthal, A.S.: Order-picking in a rectangular warehouse: A solvable case of the travelling salesman problem. Operations Research 31(3), 1983, S. 507-521.

Roodbergen, K.J. und de Koster, R.: Routing order pickers in a warehouse with a middle aisle. European Journal of Operations Research 133, 2001, S. 32-43.

Ruben, R.A. und Jacobs, F.R.: Batch construction heuristics and storage assignment strategies for walk/ride and pick systems. Management Science 45(4), 1999, S. 575-596.

Dieser Artikel entstand im Rahmen des von der DFG geförderten Forschungsvorhabens « Blockiervorgänge in manuellen Kommissioniersystemen mit statischer Artikelbereitstellung » (Geschäftszeichen FU-273/4-1) 